\title{
Interactive Multidimensional Display of Magnetic Resonance Imaging Data
}

\author{
Derek Ney, Elliot K. Fishman, and Leonard Dickens
}

\begin{abstract}
Acquisition of multiparametric images in multiple planes often requires unacceptably long scanning times. The ability to display high quality planer cuts in arbitrary planes from single plane leg, transaxial, coronal, or sagittal planel images would alleviate the need to acquire images in multiple planes. The need to display data from three-dimensional volume acquisitions also poses a problem to the radiologist. We have developed an interactive multidimensional display tool for magnetic resonance data. The tool presents three orthogonal planes (such as transaxial, coronal, and sagittal) simultaneously and allows the user to interactively roam through the data set. The user can select any arbitrary oblique plane and obtain the corresponding reformations. Additionally the tool allows the correlated display of sets of differently acquired data. This tool offers an effective means for the display of isotropic data and reformatted planar data. The ability to interact directly with the data allows increased transference of information to the radiologist and referring physician.

(C) 1990 by W.B. Saunders Company.
\end{abstract}

KEY WORDS: magnetic resonance imaging, image processing, multiplanar imaging.

M AGNETIC RESONANCE imaging (MRI) has become an increasingly important diagnostic tool for the radiologist and referring physician. The ability to apply differing pulse sequences allows the acquisition of images that are specifically tailored toward a specific diagnostic problem. ${ }^{1}$ Additionally, the ability to scan directly in any plane (ie coronal, oblique, etc) allows for a better understanding of structures that are not well displayed in a conventional transaxial slice. ${ }^{2}$ A newer technique, volume acquisition, ${ }^{4}$ promises to produce data with unprecedented resolution in all directions (an isotropic dataset). This should allow for increased understanding of three dimensional structures that wander in and out of imaging planes.

Unfortunately, the ability to acquire data directly in any plane does not always offer all of

From the Department of Radiology, The Johns Hopkins Medical Institutions, Baltimore, $M D$.

Address reprint requests to Derek Ney, Department of Radiology. The Johns Hopkins Medical Institutions, $600 \mathrm{~N}$ Wolfe St. Baltimore. MD 21205.

(1) 1990 by W.B. Saunders Company.

0897-1889/90/0304-0006\$03.00/0 the flexibility it appears to. One cannot realistically acquire data in all sequences and in all planes desired, due in part to considerations including long acquisition times and patient throughput. ${ }^{5}$ By utilizing multiplanar reconstruction, one is able to acquire scans in one plane, and using the computer produce planes oriented obliquely to the original scanning plane. ${ }^{6} \mathrm{We}$ have developed a program termed "OrthoTool" that simultaneously displays the original acquired plane, as well as the reformatted planes perpendicular to the scanning plane. This tool has been used very successfully with computed tomography (CT) data. ${ }^{7}$ The user can roam interactively through the volume of data in any plane as well as interactively select and generate any oblique plane desired.

OrthoTool is ideal for the display of isotropic volume acquisitions. The user can interactively roam through the entire dataset with all planes simultaneously displayed. A similar display of an isotropic dataset on film would be incredibly bulky and awkward. The OrthoTool display offers an effective and efficient means of communicating the information to the user.

This paper presents the technical aspects of multiplanar presentation of MR data, as well as a number of patient studies that illustrate the utility of this tool. Typically, these images are viewed interactively on the imaging system, or placed on videotape for later review. We find that this interactive format helps the user understand complex anatomic and pathologic data. The paper attempts to capture in print the interactive nature of OrthoTool of MRI data.

\section{TECHNIQUE}

The first step in the display process is to transfer the data from the MR scanner to the freestanding imaging system. The data was obtained on either a GE (General Electric Medical Systems, Milwaukee, WI) 1.5T Signa System or a Siemens (Siemens Medical Systems, Iselin, NJ) Magnetom 2T system. The free standing imaging system used in our work consists of a Sun $3 / 160$ or $4 / 280$ Workstation (Sun Microsystems, Mountain View, CA) with an attached 
PIXAR image computer (PIXAR, San Rafael, CA). The PIXAR image computer is a highspeed graphics accelerator. The PIXAR is used to handle all of the image processing and image display tasks. The PIXAR system used consists of 4 parallel processors and 24 Mbytes of image display memory.

The data is transferred to the imaging system via magnetic tape. During the transferring process, the volume of data is interpolated to produce volume elements (voxels) with equal dimensions in all directions (isotropic). The interpolation process is done on the PIXAR. The voxel resizing is implemented by use of a bicubic interpolation using a Catmull-Rom basis function. The bicubic interpolation uses four adjacent pixels to produce each interpolated value. (A linear interpolation uses only two adjacent pixels for each interpolated value.) If the scanned volume was obtained with an isotropic volume acquisition, this interpolation is unnecessary, because the voxels already have equal dimensions in all directions. The interpolated dataset as well as the original data are then saved to the system hard disk.

Once that data is saved, the OrthoTool program is started. It begins by loading the image volume(s) into the PIXAR's main memory. The program then displays a transaxial, coronal, and sagittal section of the volume on the image monitor screen. Corresponding three dimensional images of the volume are created for use as orientation guides in the upper right quarter of the display (Fig 2A). The user is presented with the user interface screen on the Sun workstation display shown in Fig 1. By positioning the mouse cursor on any one of the sections (transaxial, coronal, etc) and clicking the left or right mouse buttons, the user can roam through the data one image at a time. If the mouse button is held down the user rapidly advances through the data. The user can arbitrarily roam through any of the three displayed planes of data. To select an oblique slice (nontransaxial, coronal or sagittal) the user simply draws a line on any one of the display planes. The oblique button is then pressed and the entire volume is rotated so that the specified plane is displayed. The other two planes displayed are mutually orthogonal to the specified plane (Fig $4 \mathrm{~A}$ and $\mathrm{B}$ ). The oblique transformation process takes approximately ten seconds. The user can also make linear and angular measurements on any of the planes (Fig $2 \mathrm{~A}$ ).

The OrthoTool program can also display multiple spatially correlated sequences of the same patient. For example a multiple echo study may be acquired such that two datasets are pro-

Fig 1. The user interface screen for OrthoTool. The controls in the upper right of the large window are used to manipulate the volume. The blank area around the controls is used for roving the cursor. The control window at top is used to manipulate window width and lovel.

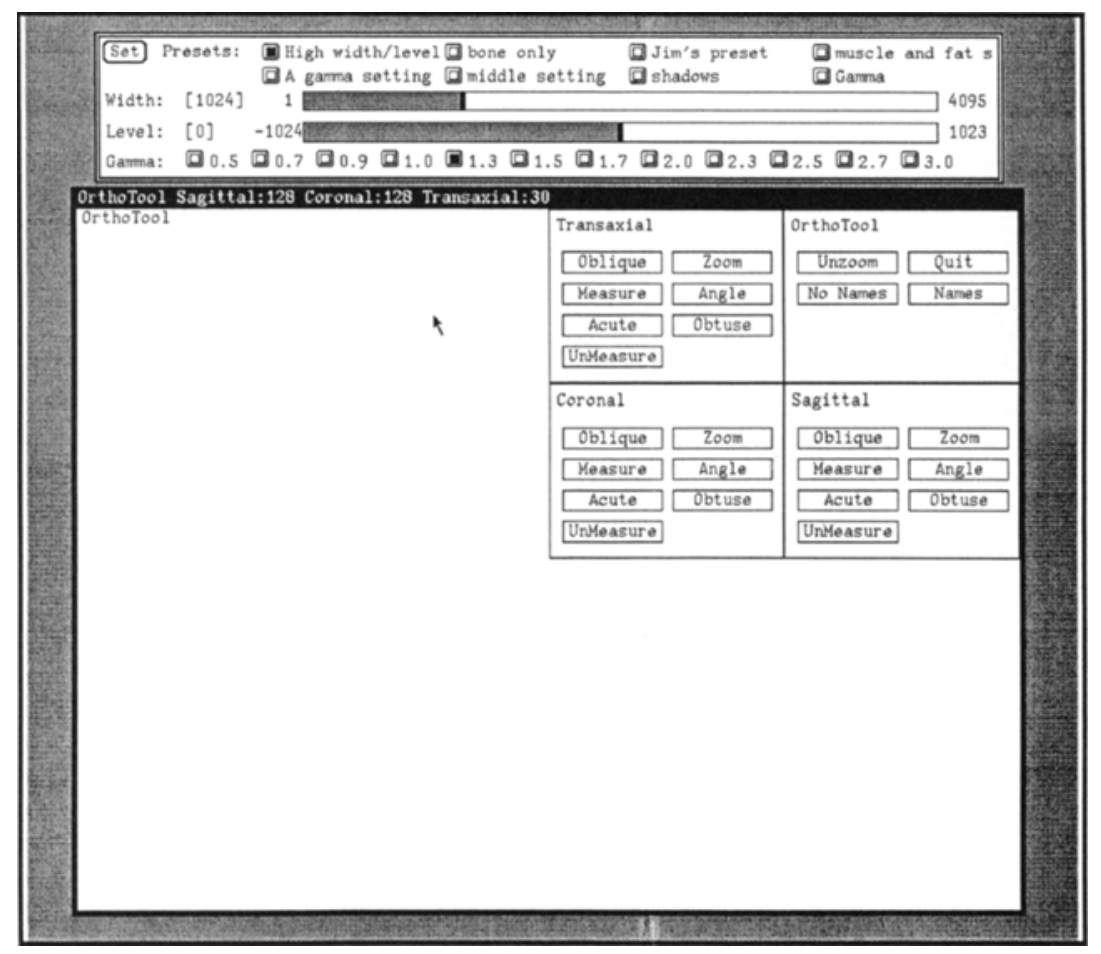



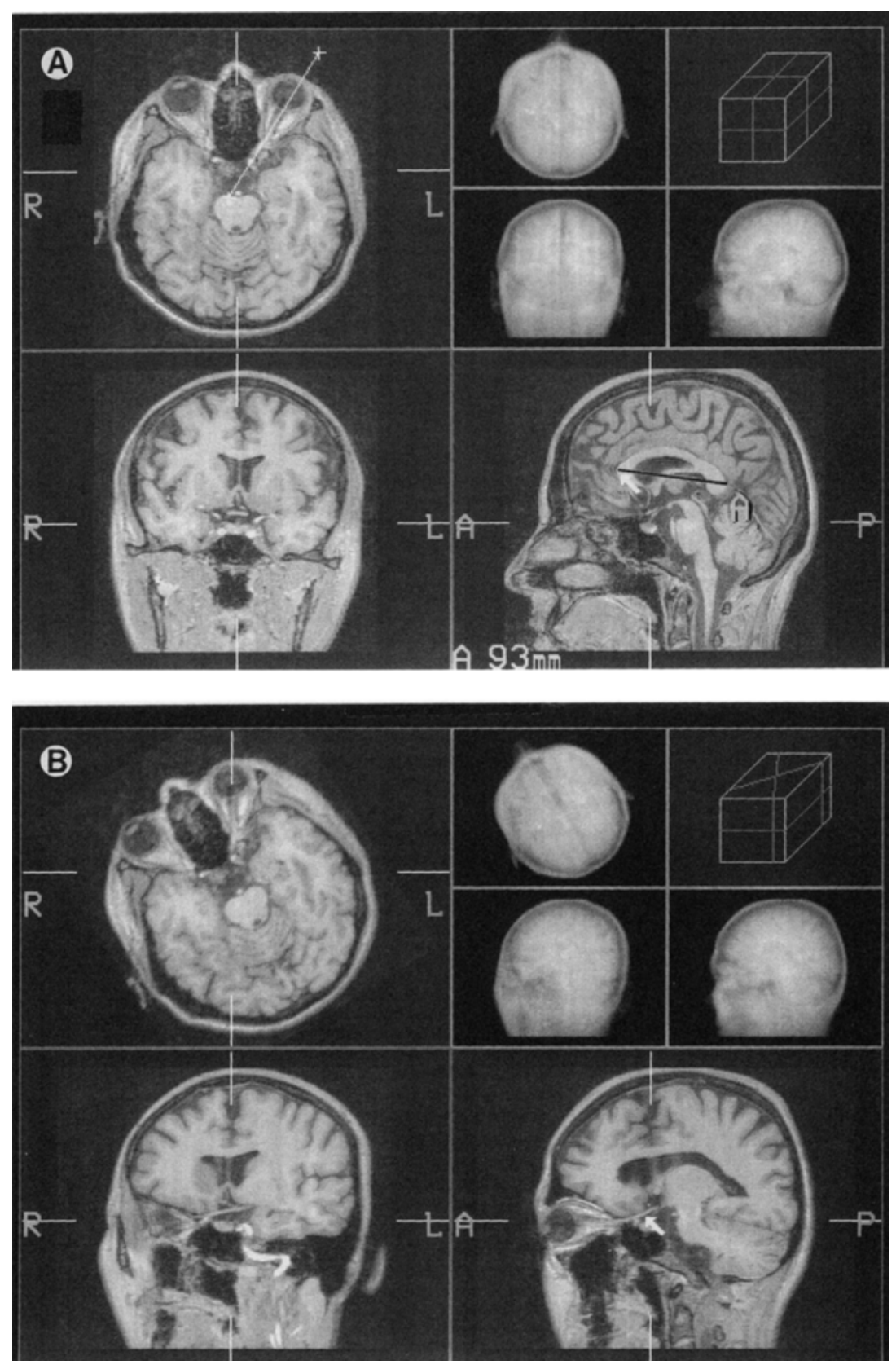

Fig 2. (A) images from a FLASH valume ecquis|tion of a normal brain. Transaxial, coronel, and sagittal sections are displayed. Note the linear measurement of ventricular depth. The images in the upper right are three dimensional projection Images of the volume used for orientetion purposes. (B) Display of arbitrery oblique slices through the optic nerve (arrow). The user can roam through these oblique sections interactively. duced-a sequence with parameters of echodelay time (TE)/pulse-repition interval (TR) $20 / 2000$ and TE/TR $80 / 2000$ milliseconds. These sequences can be simultaneously displayed. ${ }^{8,9}$ Typically, each sequence is assigned a certain color mapping so that when combined, a super image shows both sequences such that easy correlation can be made between the sequences. Currently, memory limitations allow only three sequences to be displayed, and arbitrary oblique selection cannot be made. The addition of 12
Mbytes of memory would allow for the display of up to six sequences and arbitrary oblique sectioning.

When two sequences are displayed, one sequence is mapped to red plus half green (light yellow) while the other sequence is mapped to blue plus half green (aquamarine). When combined, areas in the image that have equal values for each sequence will appear as varying intensities of white. Unequal areas will be displayed as colored areas. When three sequences are dis- 


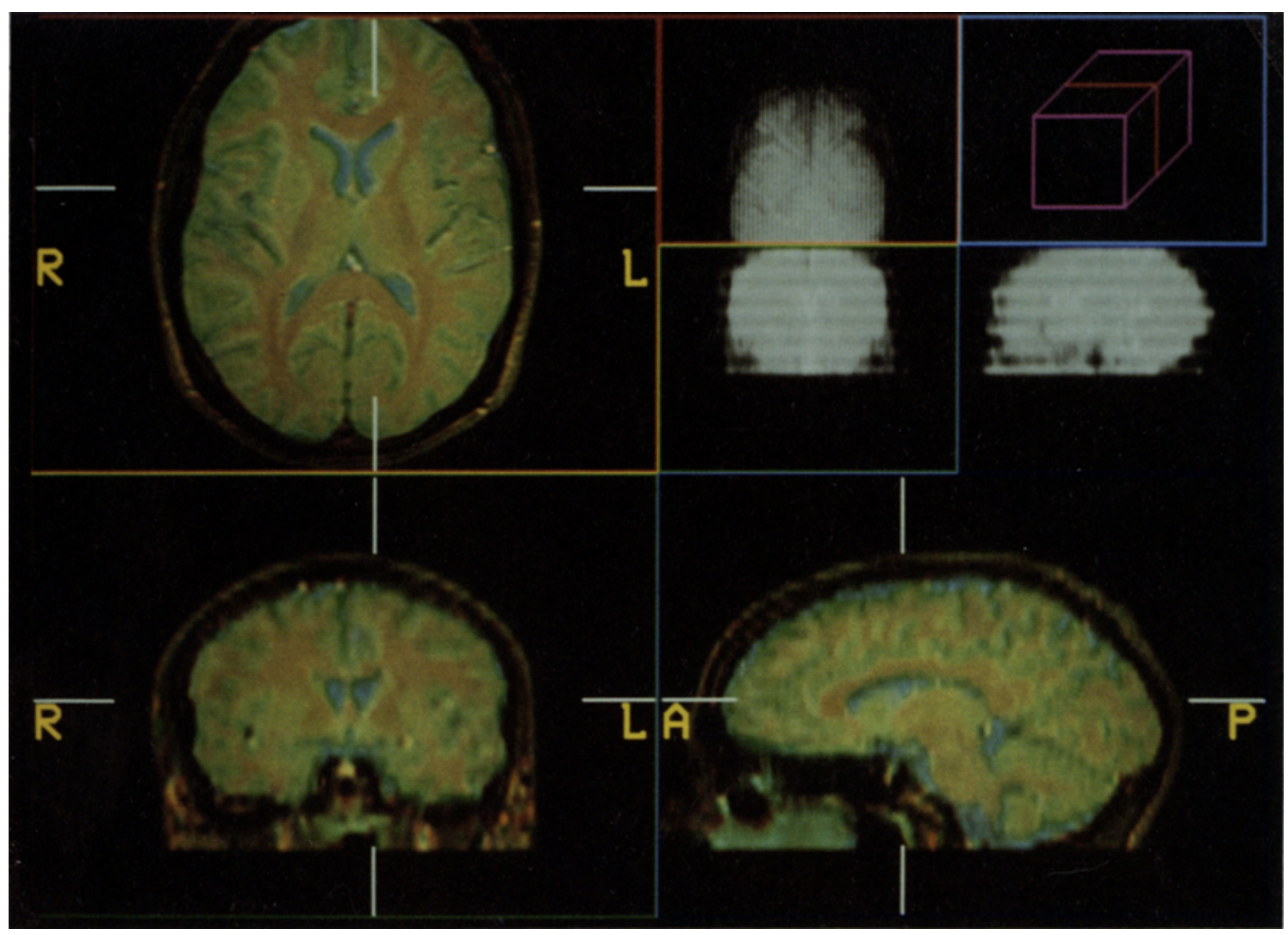

Fig 3. Correlated display of multiple sequences acquired of a normal brain. Three sequences are displayed: a T1, a T2, end a spin density weighted sequence. The T1 sequence is assigned red, the spin density to green and the T2 to blue. Images were acquired in the transaxial plane and reformatted to produce the coronal and sagittal planes.

played, one image is mapped to red, one mapped to green, and one mapped to blue. When these images are overlaid, equal areas in all three sequences are displayed as varying shades of white. Unequal area is colored appropriate to the different values of a voxel in each of the three sequences.

Due to the limited amount of memory available on the system, when multiple sequences are displayed, the uninterpolated data (with unequal dimension of each side of a voxel) is loaded into image memory. During the display of one given plane from the volume, the image is resized using a linear interpolation to obtain the correct aspect ratio. The displayed image will appear the same as if the interpolated data were used. The use of the uninterpolated data allows the display of larger volumes and more sequences than the use of the interpolated volumes.

For clinical use, the OrthoTool image display is videotaped on $3 / 4$ in or VHS tape as the user roams the dataset. This videotape can then be sent to the referring physician. This makes for a cost effective, convenient method of transference of information. In some cases the referring physicians will come to the system to view the images directly. Additionally, a multiformat camera can be used to make prints of the OrthoTool display.

\section{CASE STUDIES}

\section{Case I}

The first case is a volume acquisition of a normal brain. The scans were acquired on a Siemens Magnetom 2T scanner with a Flash 40 protocol. Parameters were TR of 0.04 milliseconds, TE 5.0 milliseconds, producing 116 sagittal slices $(256 \times 256)$. The data was transferred to the imaging system and displayed using OrthoTool. Fig 2 A shows representative images obtained by roaming through the volume. Fig 2 B shows an arbitrary oblique cut through the optic nerve. Note the high quality of the resulting images.

\section{Case 2}

$T 1, T 2$, and spin-density-weighted images of a normal brain were acquired. Images were obtained on a GE Signa $1.5 T$ system. Parameters for the T1-weighted study were TE 

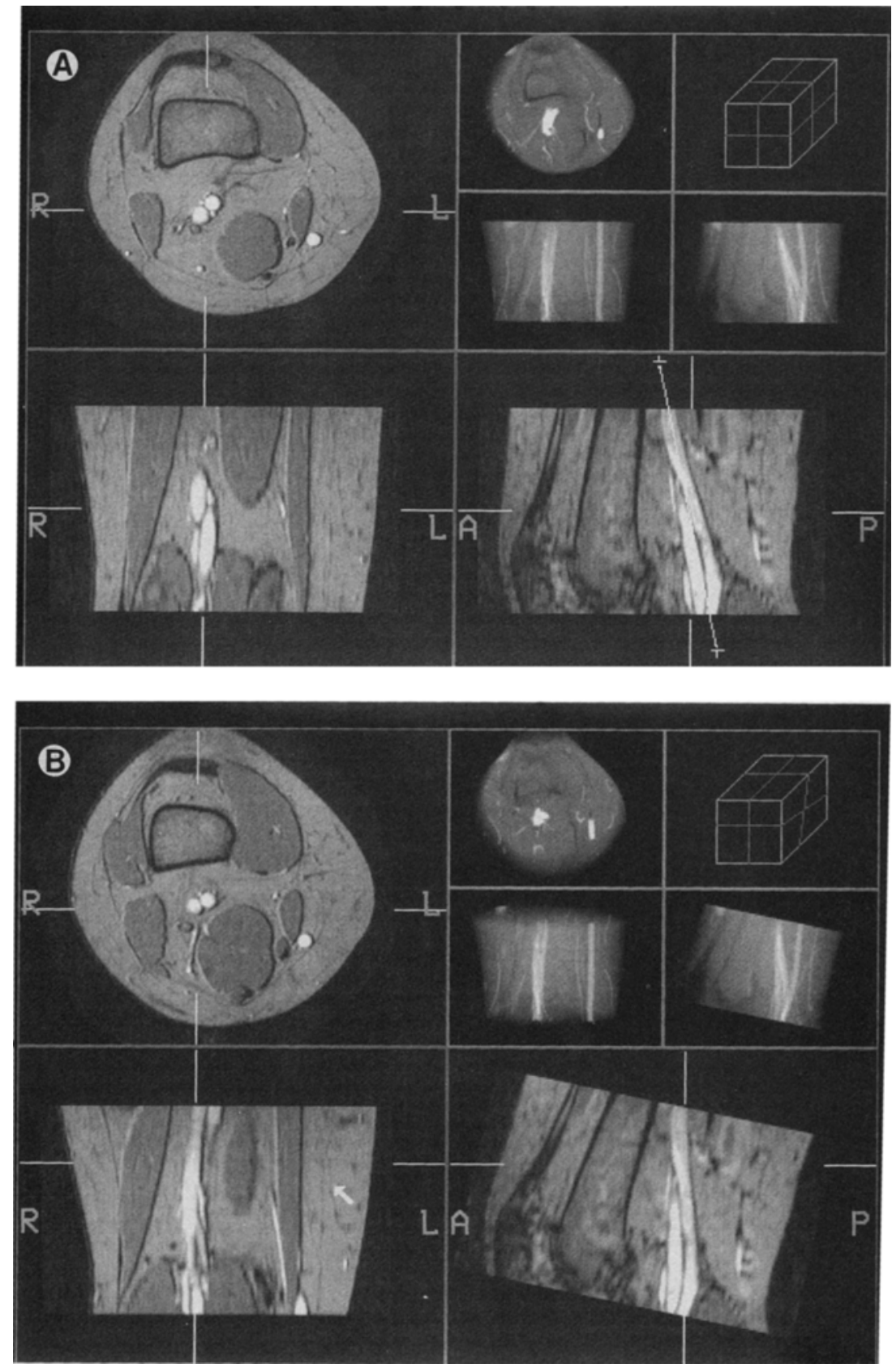

Fig 4. (A) Images from a gradient refocusing acquisition of the normal knee. Vessels are segn clearly in these images. The line shows the selection of an arbitrary oblique slice displayed in Fig 4B. (B) An oblique section through the knee illustrating the popliteal artery and vein. The ability to interactively select an oblique plane allows for the optimal display of out-ofplane structures.
30 milliseconds, TR 600 milliseconds. Parameters for the T2-weighted study were TE 80 milliseconds, TR 2,500 milliseconds. Parameters for the spin-density weighted study were TE 30 milliseconds, TR 2,500 milliseconds. All slices were $5 \mathrm{~mm}$ thick with a resolution of $256 \times 128$, and were acquired in an interleaved fashion such that contiguous slices resulted. The images were then colored so that the T1weighted scans were assigned red, the spin-density-weighted scans assigned green, and the T2-weighted scans assigned blue. Fig 3 illustrates this case. The ability to show the three sequences in an overlapping fashion allows the physician to directly correlate findings from the three sequences. This direct correlation has obvious benefits over mental correlation of three images displayed side by side. The display of overlapped images can allow for better visualization of small signal variation from one pulse sequence to another. Small features are much harder to correlate mentally, and so can be enhanced by computer aided overlap of images.

\section{Case 3}

Gradient refocusing images were obtained of a normal knee. The study was acquired on a GE Signa $1.5 \mathrm{~T}$ scanner. Parameters were FOV $20 \mathrm{~cm}(256 \times 256)$, TR $67 \mathrm{~ms}$, TE 
Fig 5. Three dimensional image of the surface of the brain. Images were generated using the volumetric rendering technique. Images are from the data shown in case 1.

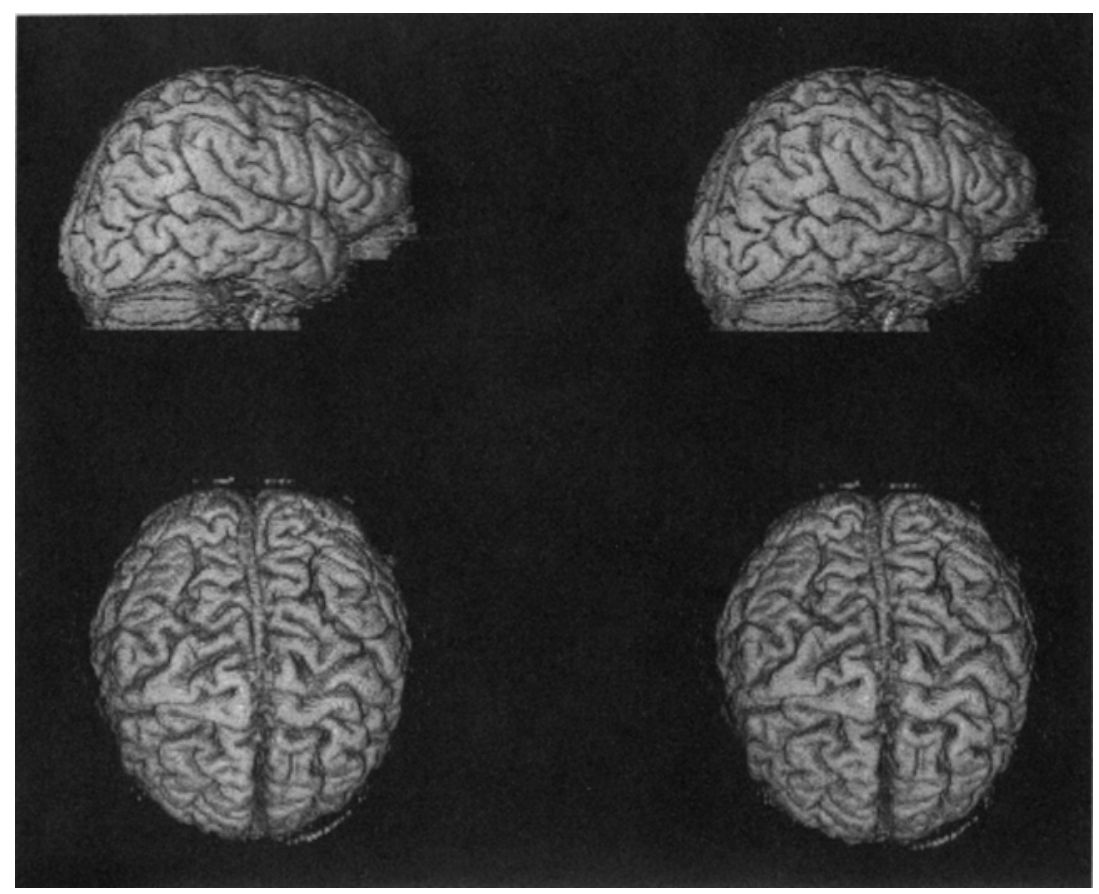

$15 \mathrm{~ms}$, and flow compensation. The slices were $3 \mathrm{~mm}$ thick. This sequence enhances blood flow. The line drawn on the sagittal image (Fig 4 A) shows selection of an oblique slice through the popliteal artery and vein. The ability to choose arbitrary oblique planes allows the display of vessels that do not lie in a standard imaging plane. Fig $4 \mathrm{~A}$ and $4 \mathrm{~B}$ illustrate the case.

\section{DISCUSSION}

The method of display of MRI data is an important consideration in any clinical department. Although display of acquired slices on film is an effective means for diagnosis, other techniques offer increased ability for display of out of plane structures and communication of results to the referring physician. Additionally, the display of volume acquisitions on film tends to be unwieldy as well as incomplete. Conventional displays cannot effectively display multiple correlated sequences. The OrthoTool displays computed multiplanar reformations of the original planar slices. It allows the overlapping display of multiple correlated sequences. It is ideal for the interactive display of volume acquisitions. The OrthoTool also gives the user the opportunity to interactively select oblique planes which best show the structures of interest.

Future developments to the OrthoTool program will allow for the correlated display of MRI data with other modalities such as CT, positionemission tomography (PET), and single photon emission computer tomography. ${ }^{10,11}$ This correlation will form a "super modality" that should offer increased information for oncologic study and other diseases. 3D imaging of MRI data is also possible using volumetric rendering. ${ }^{12-14} \mathrm{Fig}$ ure 5 shows 3D images of the study illustrated in case one. 3D imaging used in conjunction with multiplanar imaging should aid in clinical management of complex cases. Some studies have shown that by mapping functional images such as PET onto the 3D images allows for very accurate presurgical planning. The images also have potential application in many research areas. The detail of the sulcal and gyral anatomy allows the localization of functional areas of the brain from MRI data. We feel that the display of planar slices together with 3D images forms an ideal clinical study with the maximal amount of information transmitted to the clinician.

\section{CONCLUSION}

The OrthoTool is ideal for the display of MRI data. The display of high-quality multiplanar reconstruction potentially will result in a reduced need for a large number of studies acquired in 
different planes for a particular case. With the high cost of MR imaging equipment, cost effective imaging is becoming of even greater importance. Furthermore, the display of volume acquisitions can be done effectively only with a tool such as OrthoTool. Effective communication of imaging data to the user is an important part of the imaging process. Using OrthoTool with MRI data facilitates this communication.

\section{ACKNOMEDGMENTS}

We wish to thank Dr. Elliot McVeigh, Dr Elias Zerhouni, Dr Gerhaud Laub, and Siemens Medical Systems for acquiring the image data used in this paper.

\section{REFERENCES}

1. Paling MR, Abbitt PL, Mugler JP, et al: Liver metastases: Optimization of MR imaging pulse sequences at 1.0T. Radiology 167:695-699, 1988

2. Steiner RE: Magnetic resonance imaging: Its impact on diagnostic radiology. AMJ Roentgenol 145:883-893, 1985

3. Wedeen VJ, Chao YS: Rapid three-dimensional angiography with undersampled MR imaging. J Comp Assist Tomogr 11:24-30, 1987

4. Bradley WF Jr: Comparing costs and efficacy of MRI. AMJ Roentgenol 146:1307-1310, 1986

5. Fishman EK, Magid D, Mandelbaum BR et al: Multiplanar (MPR) imaging of the hip. Radiographics 7-54, 1985

6. Ney DR, Fishman EK, Magid D, et al: Interactive real-time multiplanar CT imaging: The 2D-3D Orthotool. Radiology 170:275-276, 1989

7. Ortendahl DA, Hylton NM, Kaufman L, et al: Signal to noise in derived NMR images. Magn Reson Med 1:316338,1984
8. Ottendahl DA, Hylton NM, Kaufman L et al: Analytical tools for magnetic resonance imaging. Radiology 153:479488,1984

9. Dickens L, Ney DR, Fishman EK, et al: Multidimensional simultaneous display and analysis of SPECT and CT data. Radiology 173:49, 1989

10. Levin DN, Pelizzari CA, Chen GTY, et al: Retrospective geometric correlation of MR, CT and PET Images. Radiology 169:819-823, 1988

11. Drebin RA, Carpenter L. Hanrahan P: Volume rendering. Computer Graphics SIGGRAPH 1988 Proceedings 22:65-74, 1988

12. Ney DR, Fishman EK, Zerhouni EA, et al: Three dimensional volumetric image processing applied to MR Imaging of the Liver. Radiology 165:202, 1989

13. Levin, DN, Hu X, Tan KK, et al: Surface of the brain: Three-dimensional MR images created with volume-rendering- Radiology 171:277-280, 1989 